\title{
Perceptions of Health Warning Signs in Seriously Ill Woman of Childbearing Age in Kinshasa
}

\author{
Thérèse Nyangi Mondo Mambu ${ }^{1}\left(\mathbb{0}\right.$, Patrick Kalambayi Kayembe ${ }^{1}, M^{2}$ riam Malengreau², \\ Bruno Dimonfu Lapika ${ }^{3}$
}

${ }^{1}$ Faculty of Medicine, Kinshasa School of Public Heath, University of Kinshasa, Kinshasa, Democratic Republic of the Congo

${ }^{2}$ School of Public Health, Catholic University of Leuven, Brussels, Belgium

${ }^{3}$ Department of Sociology and Anthropology, University of Kinshasa, Kinshasa, Democratic Republic of the Congo

Email: mambutlm2@gmail.com

How to cite this paper: Mambu, T.N.M., Kayembe, P.K., Malengreau, M. and Lapika, B.D. (2021) Perceptions of Health Warning Signs in Seriously Ill Woman of Childbearing Age in Kinshasa. Health, 13, 886-902.

https://doi.org/10.4236/health.2021.138068

Received: June 26, 2021

Accepted: August 27, 2021

Published: August 30, 2021

Copyright ( 2021 by author(s) and Scientific Research Publishing Inc. This work is licensed under the Creative Commons Attribution International License (CC BY 4.0).

http://creativecommons.org/licenses/by/4.0/

(c) (i) Open Access

\begin{abstract}
Introduction: For seriously ill women of childbearing age, perceived health warning signs may influence decision of whether or not to seek care. Inaccurate perceptions of patients and those around them may lead to attitudes that delay seeking care. This study analyzes perceptions of danger and related delays to seek care in Kinshasa. Methods: Sixty deceased women who died between March and April 2004 were taken away from two Kinshasa mortuaries. History of disease and deaths were reconstructed through medical records and semi-structured interviews of family members and leaders. The Qualitative Software Research was used to conduct a qualitative analysis. Results: Perceived health warning signs had five manifestations: Specific clinical health warning signs, aggravation of non-specific signs, persistence of signs, indirect danger signs and superstitious signs. The incorrect perception of the signs was an important cause of late awareness of the danger and delayed decision to seek care. Conclusion: Misunderstandings of signs often delayed awareness of danger as well as decisions to seek appropriate care. Educational programs teaching health warning signs should be designed to promote the timely use of facilities.
\end{abstract}

\section{Keywords}

Perception, Health Warning Signs, Seeking Care, Kinshasa

\section{Introduction}

The World Health Organization (WHO) estimates that, worldwide, more than 500,000 women annually die from causes related to pregnancy and childbirth, 
$40 \%$ of which are from Africa, particularly sub-Saharan Africa [1]. The mortality of women of reproductive age, those between 15 - 49 years of age, is a public health priority in developing countries and especially in sub-Saharan Africa. Research on women's health in developing countries should not be limited to estimating mortality and morbidity; it should also seek to understand healthcare utilization among women [2]. Perceptions of signs of illness are a key element in initiating the process of seeking care [3]. The degree to which symptoms interfere with lifestyle and daily activities may ultimately compel a woman to seek care [4].

In the Democratic Republic of Congo (DRC) in 1998, the national survey "State of Health" directly estimated the maternal mortality rate to be 1837 deaths per 100,000 live births [5]. In 2001, another national survey, Multiple Indicators Cluster Sample Survey 2 (MICS2), which used a "sister method" which is an indirect method, estimated maternal mortality rate to be 1289 per 100,000 live births [6]. The 2007 Demographic and Health Survey estimated it to be 549 maternal deaths and more recently that of 2014 estimated it to be 846 deaths per 100,000 live births [7] [8]. Regardless of the differences in the methodological approach of these surveys, what remains clear is that many women of childbearing age die from preventable causes [9] [10] [11]. One of the factors contributing to these deaths, a significant proportion of which is preventable, is the delay in seeking health care due to misperceptions about health Warning Signs [11] [12].

Heath Warning Signs considered by the patient and those around her can be either physical (material) or cultural. To fully understand the journey through all stages of an individual's quest for care, it is necessary to understand how she and those around her interpret and assess symptoms and how they seek care [13]. The purpose of this study is to describe the different ways that women perceive heath warning signs as well as the attitudes they adopt after they notice symptoms of severe diseases.

\section{Methods}

\subsection{Types of Study}

To describe health warning signs and common perceptions of them, a descriptive study using qualitative approach by using semi-structured interviews to collect stories about illness and death was conducted. The study sought parents of women of childbearing age who have died or any adults close to the deceased who witnessed the course of their diseases. To correct the subjectivity of the affected families, the data collected from families was cross-checked with that of community leaders ${ }^{1}$ to observe typical attitudes and behaviors towards diseases.

${ }^{1}$ Community leaders were chosen from among men or women who had a role that enabled them to have relevant information about the community. These last were dynamic characters in regular contact with all events in the community because of their extroverted position or their profession which required them to regularly visit households in their neighborhood. They were street chief, neighborhood chief, presidents of socio-cultural associations, religious associations, pastors, members of the health committee, basic cell head of the Catholic Church, nurses, teachers, leaders of revival churches and notables. The list was kept open because it could vary from one district to another. 
This cross-checking with community leaders also made it possible to increase the validity of the results obtained through the triangulation of sources [14]. The information collected focused on health warning signs for each case, the attitude adopted after their occurrence, possible delays caused in the event of incorrect understandings of the signs, and the evolution of disease symptoms. First, confirm that you have the.

\subsection{Cadre of Study}

The cases analyzed in this study were taken from two morgues in the city of Kinshasa, the capital of the DRC. Kinshasa is a city of nearly 10 million inhabitants and had four main morgues (at the time of the investigation) which received corpses of all categories socioeconomic and other medical centers. These included the Central Municipal Morgue of Kinshasa, the morgue of the Bondeko Clinic, the morgue of the University Clinics and that of the Ngaliema Clinic. Data from two of these main morgues, namely the first two (the Central and that of Bondeko), were used for this study.

\subsection{Definition of Keys Concepts}

Health Warning Signs refer to any symptom or physical sign that may be caused by a life-threatening illness if prompt medical intervention is not conducted. Due to the difficulties inherent in this context, this study is limited to cases in which the patient survived for at least 24 hours after the onset of danger signs.

A serious case refers to any pathology in a patient that can cause severe symptomatology which can subsequently put her life in danger. This case would require immediate effective medical care because the patient's condition would be considered life-threatening.

There is a delay in seeking care when the interval between the discovery of the first signs of danger and medical treatment at the suitable level is marked by a delay which may be partly responsible for the death of the patient. [15]

Delay in seeking care may consist of a few varieties, such as delay in awareness of danger (when the time between the appearance of danger signs and awareness is at least two days), delay due to the attribution of the disease to a cultural cause or due to the use of alternative care (when the time spent with the traditional healer, prophet, or pastor is more than a day), delay in decision to seeking care (when the time between awareness of the danger and the execution of the remedy is greater than two hours), delay linked to difficulties in directing the patient to the appropriate services (when the time interval from the execution of the remedy until the access to the appropriate place of care is greater than two hours), delay in taking charge (when the time due to the unavailability or incompetence of the nursing staff exceeds one hour), and delay in administering treatment (when the time from the prescription of treatment until execution exceeds one hour) [11].

Incorrect perceptions concerning symptoms of a disease may take the form of 
non-recognition or underestimation of signs of danger and disease; these perceptions may also involve attribution to a trivial cause or concealment of the perceived signs. Misinterpretation of signs often indicates alternative care, which may explain the absence or delay of appropriate treatment.

\subsection{Selection of Participants, Data Collection and Processing}

As the study did not aim to be exhaustive or to provide quantitative measures, the cases studied were selected on the basis of the information they could provide and not based on their representativeness of all categories of the population. The deceased women in this study were selected from the Kinshasa Central Morgue and the Bondeko Clinic Morgue, which are the two main morgues in the city and which hold bodies from all socioeconomic categories and from other hospitals. All women aged 15 to 49 years who died between March and April 2004 and whose bodies were deposited in these two morgues were taken into account, i.e. 275 women aged 15 to 49 years out of a total of 1,383 deceased persons registered during this same period. The collection took place between June and October 2004. The interviews took place between 3 and 6 months after the death.

Of these 275 women, we excluded 5 indigent women living alone for whom there was no fixed address and therefore no respondents. Of the remaining 270 women, 152 died in a medical facility and 118 died at home or on the road. We excluded 4 women whose death occurred less than 24 hours after the onset of the first danger signs and 57 women who died of known incurable causes (terminal cancer, decompensated cirrhosis, terminal heart disease, pancreatic cancer, or end-stage AIDS) with no possibility of treatment in the DRC, Wessel et al. used the same approach in their study of factors of death of women in Cape Verde [9].

Of the 209 cases remaining out of 275, thirty-four cases were discarded for various reasons: 21 non-preventable cases, 2 non-existent addresses, one woman died at 96 years of age, one deceased was a boy, one woman died outside of Kinshasa, and in 9 cases there were no valid informants. Of the remaining 175, we undertook the interviews commune by commune and were able to visit only 101 households in eleven of the twenty-four communes in Kinshasa. Sixty-six households met all the conditions (age, existence of address, preventability of death, family member available for interview, and death occurring at Kinshasa) and 60 interviews were completed, the other 6 having refused to participate. This allowed us to conduct 60 detailed interviews. The number of 60 seemed sufficient to identify the diversity of circumstances of death, attitudes and behaviors as well as the routes taken before death, and the main explanations for delays in seeking medical care in case of danger. The interviewee was selected from among adults who had assisted the deceased or lived regularly with her, if not among those who observed and followed the course of events during the illness.

The history of illness and death for each woman was reconstructed on the basis of available medical records and semi-structured interviews conducted with family and friends, two to six months after the woman's death. The information 
obtained was then cross-checked or supplemented with that of leaders of the community where the woman resided at the time of her death. The interviews were carried out by a female doctor accompanied by a sociologist, who took notes; the interviews were recorded and transcribed before being supplemented with interviews of community leaders. The questions for this study and the narrative analysis focused in particular on the perception of signs of illness and causes, as well as attitudes and behaviors in the presence of health warning signs.

To establish the cause of death, and to estimate the extent to which death could have been prevented by timely medical care, we first reviewed 50 medical records of women who died in a medical facility and completed 50 data sheets from them. These records were selected on the basis of their availability at the facility level and the presence of the information sought and were therefore not representative, but only illustrative. For 16 women who had died at home and on the road, we used the data collected during the interview with the family and friends to complete our data collection forms. The 50 data sheets collected from the medical records and the 16 sheets for women who died at home were grouped according to their municipalities of residence before death. Probable causes of death were estimated on the basis of the direct cause noted in the medical record (when there was one) or the one estimated independently by two physicians (including the investigator) from information collected from the family and leaders.

To interview one or two leaders from the communities affected by each death, we visited 99 households in which 64 interviews were completed. Five leaders were excluded because they were too involved in the death (family members or close friends), 3 refused to be interviewed, 25 did not respond to the request for an appointment, and 2 interviews were interrupted due to fatigue.

Statistical Package for Social Science (SPPS), quantitative analysis software was used to describe the socio-demographic characteristics of the sample and respondents. The qualitative analysis software: Qualitative Software Research, Non Unstructured Data Indexing and Theorizing 6 (QSR NUD IST6), which can be classified as "hypothesis-generating" software, was used to process the content of the interviews, identify the ideas contained in each theme by inductive approach, and then classify and associate the different perceptions, attitudes, and practices so that they could be illustrated by excerpts from interviews [16].

\subsection{Ethical Considerations}

The data collection in this study followed ethical principles. Its protocol was approved by the ethics committee of the Kinshasa School of Public Health.

Addresses and identities were only used to locate the families of those involved in the study; afterwards, they were destroyed, and no one else had access to this data other than the researchers involved in the study. The publication featured total anonymity. There was no personal compensation for participation in the study, and the results obtained were used to support efforts leading to the reduction of mortality among women of childbearing age. Informed consent was 
obtained from family members and community leaders. They were allowed to discontinue their participation in the study as soon as they wished.

\section{Results}

\subsection{Characteristics of the Sample Studied}

\section{Characteristics of the 60 deceased women}

Out of the 60 women included in the study, the average age was 32 years and ranged from 18 to 49 years. The socioeconomic level of the households of the deceased was assessed using the Adjusted Poverty Index ${ }^{2}$ (API index), based on possession of property. According to this index, $5 \%$ lived in a poor household, $63 \%$ in a modest household, $29 \%$ in a medium or moderate household and $3 \%$ in a comfortable or affluent household. Of the 55 women whose education level was known, $48(80 \%)$ had completed some or all of secondary education, and two (3\%) were tertiary students; five (8\%) had not completed elementary school. Comparison of these data with the characteristics of the Kinshasa population in representative random sample studies [17] suggests that these women do not differ significantly in education and socioeconomic status from other women of this age in Kinshasa (Table 1) [11].

Table 1. Socio-demographic characteristics of women in Kinshasa and of women who died [11].

\begin{tabular}{ccc}
\hline Numbers & Kayembe (2000) & Mambu (2000) \\
\cline { 2 - 3 } Median age & 1148 & 60 \\
Socioeconomic level (\%) & $20-24$ & $30-34$ \\
Poor & 5 & 5 \\
Modest & 51 & 63 \\
Moderate & 38 & 29 \\
Affluent & 6 & 3 \\
Education (level started) (\%) & & - \\
None & 3 & 8 \\
Primary & 14 & 80 \\
Secondary & 74 & 3 \\
Higher & 9 & 8 \\
Unknown - & - & \\
\hline
\end{tabular}

\footnotetext{
${ }^{2}$ The Socioeconomic Level Index (SLI), API Index calculates the socioeconomic level of women based on whether or not they own certain assets such as tap water in the household, an indoor toilet, electricity, a vehicle, a television, a radio, a refrigerator, and tiles or cement in the house. The index is a composite variable calculated from the summation of assets owned. Women are classified as having "high API: well-to-do or rich" possession of all eight assets, "medium-high API: more or less well-to-do or less rich" six to seven assets, "medium API: modest" two to five assets; "low API: poor" none or only one of these assets.
} 
Of the 59 women whose occupation was known (one was unknown), 26 (44\%) were saleswomen of various articles, $16(27 \%)$ only took care of their household or had no particular occupation, 8 (13.5\%) were pupils or students, 4 (7\%) were seamstresses or braiders, $3(5 \%)$ were managers in a state enterprise, one was a currency trader and one was a musician (Table 2).

Of the 60 women who died, 15 (25\%) lived with their husbands, 10 (16.7\%) with a partner and $24(40 \%)$ did not live with their partner but resided with their parents at the time of death, 10 divorced, one widow and 13 single mothers (21.7\%); this was also the case for 11 single women (18.3\%).

With regard to the proven or presumed causes of death, 18 cases (30\%) died of AIDS sometimes associated with tuberculosis, 16 cases (27\%) of complications of pregnancy or childbirth, 12 cases (20\%) of various acute infections, seven cases (13\%) of tuberculosis and seven (13\%) of other causes (Table 2).

Characteristics of respondents

Table 3 presents the characteristics of the respondents who provided information for their deceased relatives. Sixty-eight percent of respondents were

Table 2. Sociodemographic and clinical characteristics of deceased women.

\begin{tabular}{|c|c|c|}
\hline & Deceased females aged 15 - 49 years & \multirow{2}{*}{ Percentage } \\
\hline & Numbers (60) & \\
\hline \multicolumn{3}{|l|}{ Occupations of deceased } \\
\hline Miscellaneous saleswomen & 26 & 44 \\
\hline Housewives or no occupation & 16 & 27 \\
\hline Pupils/students & 8 & 13 \\
\hline Seamstresses/Braiders & 4 & 7 \\
\hline Executives in state enterprises/companies & 3 & 5 \\
\hline Musicians/Currencytrader & 2 & 3 \\
\hline Occupations not known & 1 & 2 \\
\hline \multicolumn{3}{|l|}{ Family status and residence } \\
\hline Living with their husbands & 15 & 25 \\
\hline Lived in a common-law relationship & 10 & 16.7 \\
\hline Divorced and returned to parents & 10 & 16.7 \\
\hline Widowed and living with parents & 1 & 1.7 \\
\hline Single mothers in parents' home & 13 & 21.6 \\
\hline Single in parents' home & 11 & 18.3 \\
\hline \multicolumn{3}{|l|}{ Diseases that caused death } \\
\hline AIDS/AIDS + Tuberculosis & 18 & 30 \\
\hline Tuberculosis & 7 & 11.6 \\
\hline Acute infections & 12 & 20 \\
\hline Pregnancy-related complications & 7 & 11.6 \\
\hline Complications around childbirth & 9 & 15 \\
\hline Others causes & 7 & 11.6 \\
\hline
\end{tabular}


Table 3. Characteristics of respondents.

\begin{tabular}{|c|c|c|}
\hline \multirow{3}{*}{ Effectives } & \multicolumn{2}{|c|}{ Gender } \\
\hline & Males & Females \\
\hline & (19) & (41) \\
\hline \multicolumn{3}{|l|}{ Age (Age group) } \\
\hline $16-24$ years & 1 & 6 \\
\hline $25-34$ years & 4 & 10 \\
\hline $35-44$ years & 4 & 12 \\
\hline $45-54$ years & 1 & 5 \\
\hline $55-64$ years & 5 & 8 \\
\hline $65-74$ years & 4 & 0 \\
\hline \multicolumn{3}{|l|}{ Family relationship with the deceased } \\
\hline Brothers, Sisters & 4 & 16 \\
\hline Fathers, Mothers & 6 & 10 \\
\hline Husbands, Cohabiting Partners & 5 & 16 \\
\hline Daughters, Sons & - & 2 \\
\hline Cousins, Brothers-in-Law, Fathers-in-Law & 4 & \\
\hline Grandma, Daughters-in-Law, Niece & & 3 (1 of each) \\
\hline Aunts, Sisters-in-Law, Neighbors, Cousins & & 12 (3 of each) \\
\hline \multicolumn{3}{|l|}{ Degree of Responsibility of the Respondent } \\
\hline Responsible parent & 11 & 16 \\
\hline Primary caregiver & 9 & 22 \\
\hline
\end{tabular}

women 16 to 70 years old (with an average age of 43 ). This was most often (72\%) a first degree relative of the deceased (father, mother, brother, sister, spouse, son, or daughter). In $52 \%$ of families, the respondent was the primary caregiver.

\subsection{Perception of Health Warning Signs}

Perceived Health Warning Signs were expressed in five ways:

- Clinical signs of immediate danger: breathing difficulties, disturbances of consciousness, convulsions, vague or elusive gaze, eyes rolled back and staring at the ceiling. Here are the words of the deceased's sister, primary care giver: “... We saw her condition changed. She started to convulse, she was having difficulty breathing, and her eyes rolled back and stared at the ceiling." The community leader interviewed confirmed the comments and admitted that she had a fever, subsequently convulsed; they self-medicated for two days and went to hospital late after the illness worsened.

- Worsening of non-specific signs of diseases, such as asthenia and weight loss. Here is what a mother, caregiver and responsible for the deceased told us:

“... I was afraid of her weight loss, and my daughter told me that her body was getting weaker and weaker. This saddened us because it is a sign of seriousness 
that can lead to death."

The leader interviewed for this case could not tell us anything because he was travelled.

- The persistence of symptoms beyond a normal duration for minor ailments

"Then, on the 3rd day, dad said to himself that the condition was not improving, that we bring her to the Salvation Army health center..." This was confirmed by the leader who said she suffered of a fever and subsequently convulsed. He thought they started self-medicating at home for two or three days and went to the hospital late. It was a spiritual illness caused by the witchcraft of a neighbor.

- Indirect danger signs such as a pain attitude for acute pain or a foul odor for advanced infection. This is what a sister-in-law told us:

"She had a sudden pain which gave her a gait due to pain..., and she did not continue to prepare anymore"; the leader interviewed for this case believed that her illness was due to a family conflict (the intestinal loops were intertwined) because the husband did not pay the dowry...

Speaking about indirect signs of danger, an older sister told us:

"... A foul breath that resembled the smell of the dead, like someone who was already rotting."

The leader interviewed remembered the case well and said she had untreated sore throat. He thought they went to the hospital late and they lingered at lot at home. He thought the etiology of the disease was cultural, a conflict between the two families.

- Contextual and superstitious danger signs as in a case where jaundice and hematemesis from the previous day were seen as less concerning than the presence of two black cats.

The same big sister above added:

"And what scared us the most was the presence of 2 black cats, a bad omen and not the jaundice, nor the vomiting of blood from the day before..."

\subsection{Incorrect Perception of Health Warning Sings Attitudes Adopted as a Result, and Consequent Delays in Awareness}

Through different stories, we have listed the health warning signs that the deceased and those around them wrongly considered to be trivial as well as the reasons for this trivialization. A total of 43 out of 60 cases became aware of danger too late and consequently sought treatment too late. All stories illustrating an incorrect perception were classified into five categories according to the five reasons for trivialization of health warning signs, evolution of cases, and delays in awareness of danger that might result from these perceptions (Table 4). Each illustration of an incorrect perception represented one of the five main reasons for delays:

The first reason was the coexistence of problem signs or pathologies with a pregnancy which may have been linked when the diagnosis was tuberculosis on 
Table 4. Incorrect perceptions of health warning signs, attitudes adopted as a result, and consequent delays.

\begin{tabular}{|c|c|c|c|}
\hline $\begin{array}{l}\text { Different } \\
\text { illustrations }\end{array}$ & Symptoms or Signs & $\begin{array}{c}\text { Reasons for trivialization } \\
\text { and Attitudes adopted } \\
\text { when the signs appear }\end{array}$ & $\begin{array}{c}\text { Evolution/ Consecutive } \\
\text { delays }\end{array}$ \\
\hline $\begin{array}{c}\text { First } \\
\text { illustration }\end{array}$ & $\begin{array}{l}\text { Generalized furunculosis } \\
\text { Continuous weight loss, } \\
\text { hemorrhoids, } \\
\text { anal prolapse }\end{array}$ & $\begin{array}{l}\text { Coexistence of signs with } \\
\text { pregnancy, signs perceived } \\
\text { as pregnancy discomfort, } \\
\text { as attitude: Recourse to } \\
\text { traditional healers }\end{array}$ & $\begin{array}{l}\text { Aggravation, as } \\
\text { consecutive delays: } \\
\text { Delay of awareness } \\
\text { and delay due to } \\
\text { use of inappropriate } \\
\text { alternatives }\end{array}$ \\
\hline $\begin{array}{l}\text { Second } \\
\text { illustration }\end{array}$ & $\begin{array}{l}\text { Persistent temperature, } \\
\text { abdominal pain, } \\
\text { weight loss, Jaundice }\end{array}$ & $\begin{array}{l}\text { Symptoms involved } \\
\text { women continuing in } \\
\text { their occupations (School) } \\
\text { and as attitude: } \\
\text { used self-medicating }\end{array}$ & $\begin{array}{l}\text { No change, } \\
\text { weight loss and } \\
\text { Aggravation, as } \\
\text { consecutive delays: } \\
\text { Delay of awareness of } \\
\text { danger and delay due to } \\
\text { use of inappropriate } \\
\text { alternatives of care }\end{array}$ \\
\hline $\begin{array}{l}\text { Third } \\
\text { illustration }\end{array}$ & $\begin{array}{l}\text { Weakness, epigastralgia, } \\
\text { dyspnea on pregnancy }\end{array}$ & $\begin{array}{l}\text { Previous experience of } \\
\text { deceased woman or those } \\
\text { surrounding her, } \\
\text { trivialization by the husband, } \\
\text { by the patient and by the } \\
\text { grandmother (attributing } \\
\text { signs to the rise of fetus)and } \\
\text { attitude: self-medicating }\end{array}$ & $\begin{array}{l}\text { Aggravation and refusal } \\
\text { of consultation as } \\
\text { consecutive delays: } \\
\text { Delay on awareness of } \\
\text { danger and delay in } \\
\text { decision to seeking care }\end{array}$ \\
\hline $\begin{array}{l}\text { Fourth } \\
\text { Illustration }\end{array}$ & $\begin{array}{l}\text { Lower abdominal pain, } \\
\text { sudden and sharp pain }\end{array}$ & $\begin{array}{l}\text { Signs perceived as a } \\
\text { rodent snake } \\
\text { Attitude: Resort to } \\
\text { consulting traditional healers } \\
\text { on alarming symptoms }\end{array}$ & $\begin{array}{l}\text { Aggravation, as delays: } \\
\text { Delay on awareness of } \\
\text { danger and delay due to } \\
\text { use of inappropriate } \\
\text { alternatives of care }\end{array}$ \\
\hline $\begin{array}{c}\text { Fifth } \\
\text { Illustration }\end{array}$ & $\begin{array}{l}\text { Fever, headache, } \\
\text { thrill, obnubilation } \\
\text { unconsciousness, } \\
\text { Inconsistent words, } \\
\text { amnesia }\end{array}$ & $\begin{array}{l}\text { Precomatous obnubilation } \\
\text { confused by a difficult } \\
\text { awakening, as attitude: } \\
\text { passive observation at home, } \\
\text { prayer from brothers, resort } \\
\text { to an unsuitable center } \\
\text { which detained the patient. }\end{array}$ & $\begin{array}{l}\text { Aggravation, as delays: } \\
\text { Delay on awareness of } \\
\text { danger and delay due to } \\
\text { the use of an unsuitable } \\
\text { center }\end{array}$ \\
\hline
\end{tabular}

AIDS. Regarding the coexistence of signs with pregnancy, a brother of one deceased woman said:

"But before that, during pregnancy, she presented with furunculosis that we linked to pregnancy."

The leader interviewed for this case believed that the woman had weight loss and severe asthenia and that she was suffering from AIDS as a result of her sexual vagrancy behavior.

The second reason involved women continuing in their occupations and used self-medicating while the diagnosis was a stercoral fistula after an intestinal mastectomy, a mother of one deceased woman said:

"She started her illness at the beginning of September 2003 with persistent 
fever, abdominal pain, and weight loss, and she forced herself to go to school despite her fever, and we treated regularly with aspirin."

The third reason was ignorance, trivialization of the signs of disease, and the existence of a similar previous experience while decompensated gestational diabetes was diagnosed. A grandmother of one deceased woman, respondent for this case said:

“... B.'s husband came to leave her here at home because she was just sleeping and didn't want to work. On Thursday, she was whimpering, telling me that she had heat in her heart and difficulty breathing. The discomfort did not respond to a gastric bandage (she herself thought it was gastritis), and she asked that water is poured into her chest.

But I believed-and told her-it was the rise of the fetus (touching the epigastrium with the fetal hair). I have known the same case when I was pregnant with my son, the pastor since the pregnancy was in its seventh month and the heat did not end until after the childbirth..."

The fourth reason was the interpretation of the disease as a traditional disease when the diagnosis was post pelvic pain from peritonitis:

"She had a sharp pain as if she had been cut in the stomach with a razor blade, which gave her a hunched gait, coming back from the market, and she didn't continue to prepare. Our friend M. said she knew a mother who knew how to treat lower abdominal pain associated with infertility. She had concluded that it was the 'Nioka Nkau', a rodent snake thrown in the belly and which gnaws all around him and prevents the woman from conceiving". The leader interviewed for this case believed that her illness was due to a family conflict (the intestinal loops were intertwined) because the husband did not pay the dowry and they didn't make it to the hospital on time...

The fifth reason is a confusion of diagnostic, such as this case of the onset of loss of consciousness confused with a difficult awakening when the diagnosis was neuro-malaria encephalitis: "Her husband woke her up to find out how she was; she didn't answer but only opened her eyes. Her husband would therefore leave, and I would have to try to wake her up several times but without success. I would ask for the help of his daughters, but this was also in vain..."

The leader interviewed for this case said that the deceased had a disturbance of consciousness that the family brought her to a small clinic after a long time of prayer and they went late to hospital after lingered at home.

\section{Discussion}

This study shows that in Kinshasa, the decision to seek medical care in the presence of health warning signs is often delayed by misinterpretations of these signs as well as by the inappropriate use of health services and traditional healers.

\subsection{Perceptions of Health Warning Signs}

Perceptions concerning the severity of disease is an essential element in causing 
a patient to seek care, as emphasized by Afolabi et al. [3]. Among the elements which intervene in the decision to seek health care, our study illustrates how perceptions of disease and their causes, determine the degree of concern, and thus, the nature and the speed of the response to disease. An individual's level of secondary or higher education, age, multiparity, and number of antenatal consultations increased the likelihood of awareness of danger in Pembe's study in Tanzania [18]. However, for our study, the education level of women, mostly secondary (80\%) and of mostly modest socioeconomic level (63\%) did not seem to influence their perceptions of danger signs. The nature of the signs understood as clinical signs of immediate danger, contextual and superstitious signs of danger and the persistence of these signs ultimately caused concern among patients and their relatives, perception confirmed almost entirely by community leaders interviewed.

The factor which affected perception is cultural factor. Cultural causes (supernatural and traditional) are very frequently mentioned and can lead to alternative remedies, most often in parallel with a medical approach. Sometimes this delays or interferes with appropriate care, but does not prevent it. Delayed decision making is related to incorrect perception of danger signs. The decision to treat is only made when it is realized that the complaints, symptoms and signs observed are an expression of a disease [2] [4]. Among the elements that intervene in the decision to seek care, our study illustrates how the perception of the signs of illness and the causes of these determine the degree of concern, and thus the nature and speed of the reaction to the illness.

Thus, in 43 of the 60 cases we studied, incorrect perception of symptoms and signs effectively delayed the awareness of danger and, consequently, the decision to seek help. The danger signs went unnoticed, or were trivialized for several reasons.

For example, in Cape Verde, among adult women, lack of awareness of danger signs appears to be much less important than reluctance to seek medical attention because of the poor quality of care provided [9].

\subsection{Misinterpretation of Health Warning Signs}

A disease must first be perceived, and a sign of disease that is particularly prevalent in the population is generally perceived as normal, natural, and inevitable [19]. Additionally, the decision to seek treatment was generally not taken until it was realized that complaints, symptoms, and signs were the expression of a disease [2] [4]. Thus, goiter, which is very common in the northern provinces of the DRC, is often considered a sign of beauty that does not require care. Onchocerciasis nodules, widespread among the Tetela in Sankuru province in the DRC, are also considered a normal sign for any adult and are therefore not considered a condition requiring care [20]. Pregnancy is generally considered a normal event, and death during labor and childbirth is seen as inevitable. Likewise, certain health warning signs, such as edema or blood loss during pregnancy, can be trivialized 
[21] [22] [23]. Such trivialization was found in society when furunculosis was linked to pregnancy by a woman and those around her, when in reality, it was a condition caused by retroviruses. The leader of this community had suspected retrovirus in presence of persistence of fever, weight loss and sexual vagrancy behavior displayed by the patient. There was therefore a contradiction between the perception of the cause by the family and that of the leader who joined our. Epigastrium pain in a pregnant woman was mistaken as a rise of fetal while the woman had decompensated gestational diabetes. Failure to recognize health warning signs are a problem that can be encountered anywhere, including in industrialized countries [24]. In two-thirds of the accounts collected, health warning signs were not immediately recognized, which incorrectly reassured those around the pregnant women and further delayed the realization of the urgent need for care [11].

This study shows that the trivialization of the disease by the patient and those around her was due to the attribution of the disease to a cause commonly promoted by the popular imagination. Trivialization also resulted from the spontaneous disappearance of certain symptoms, which suggested that "it will pass"; the maintenance of the patient's daily activities, the attribution of the symptoms to normal discomforts of pregnancy, or even the loss of consciousness or confusion after a difficult awakening were explanations for trivialization as well [11]. In Africa, this failure to recognize health warning signs has been reported as a factor in obstetric deaths [12] [25], fatal malaria [26], cancer [27], tuberculosis [28], and in various acute pathologies [27].

In addition, failure to recognize the severity of symptoms was manifested in the concern for concealing signs in cases of induced abortions [12]. Various authors have found that alternative remedies delay medical care for illnesses attributed to cultural causes [11] [22] [29] [30] [31] [32] [33]. In half of the accounts in this study, a cultural cause was mentioned by those surrounding the deceased women; it was sometimes specific ailments (e.g., nioka kau) deemed curable by a traditional healer which the community leader attributed to a conflict between the two families, or sometimes supernatural causes. This is an interpretation accepted by the popular imagination and particularly among the Kongos (a tribe descending from the ancient kingdom of Kongo in Central Africa and which inhabits a good part of Kinshasa). This remedy could be explained by the desire to understand the meaning of the disease, the low cost of treatment, as well as the ability to overcome these ailments through alternative medicine. In Tanzania, signs of illness are interpreted according to sociocultural norms which may lead individuals to prefer the intervention of a traditional healer over that of a doctor. There is, therefore, a cultural influence in the interpretation of signs [12] [13]. In Colombian tuberculosis patients, the misinterpretation of signs was at the root of the delay in the decision to seek care; indeed, the presence of a chronic cough is culturally attributed to an advanced stage of influenza instead of a sign of Tuberculosis (TBC) [29]. Among South Africans, the sociocultural significance 
given to sexually transmitted infections in women was that her husband had sex with other women because she had not been able to satisfy him [30]. Incorrect perceptions and behaviors of the population were not the only explanations for delays in seeking care but above all the dysfunction of services [11].

\subsection{Limitations of the Study}

Because this study used a qualitative approach, it was not an exhaustive representation of how health warning signs are perceived, but it did offer certain real perceptions that exist in Kinshasa. Due to a lack of known addresses or information, the most vulnerable women, destitute or living alone, are not represented in our study. Sixty-two percent of our sample consisted of single, divorced, and widowed mothers, and $17 \%$ of the sample consisted of cohabiting women; this seems high to us although we lack comparative data to assess it. While it may be a sampling coincidence, it may also reflect the greater vulnerability of these groups [34]. Our data, although dating back to 2004, is still somewhat current because, to our knowledge, no study on the same subject has been published to date in the DRC.

In terms of internal validity, we studied deceased women rather than survivors so that the criterion of "danger" would not be debatable. Indeed, the fact that the women had died sufficiently proved that the problem was serious enough to threaten their lives, without neglecting the fact that there is also the influence of additional factors (socioeconomic status, level of education, medical coverage etc.). The retrospective nature of the study increases the risk of subjectivity and selective choice in memory (memory bias). Another cause of subjectivity might have been desire to please the investigator or to avoid judgment, especially since the search for a person responsible for another's death is part of local culture [35]. To reduce biases due to the subjectivity of the respondents, the data collected was cross-checked with that of community leaders, who are often well-informed about what is happening in families, what has been proven in our study. To minimize the risk of subjectivity to investigators, investigators' impressions and notes were compared daily to recorded interviews, and the analysis was regularly discussed with other physicians and an anthropologist.

\section{Conclusion}

This study sought to understand how misinterpretation of health warning signs may have resulted in delays in seeking medical attention. Misperceptions have negatively impacted the pursuit of appropriate care. It can be seen that the root of delays in seeking treatment was a late awareness of danger. To strengthen continuity of care, the Ministry of Health, through the health zone management teams that constitute the operational level of service delivery, should provide for greater awareness of comprehensive patient management among medical staff, which would promote patient retention in approved health care facilities and thus ensure better follow-up of their problems. In the same vein, it would also be 
necessary to revive the health notebooks and family files and to have projects to raise awareness of behavioral changes for the staff: empathy, rigor, and professional conscience. Regular contact with the health service improves communication between the two parties and thus better use of services. All reproductive health programs, non-governmental organizations (NGOs) concerned with health, and field workers must strengthen general health education of populations of all ages. They must teach entire communities through media and peer educators so that people can recognize health warning signs and identify appropriate places to seek care. They must also prepare themselves to react quickly in the event of medical and health emergencies. Taking such measures could decrease the high death rate in Kinshasa.

\section{Acknowledgements}

This research was funded by the University Development Cooperation (CUD) of the Interuniversity Council of Francophone Universities of Belgium (CIUF), as part of its support to the Kinshasa School of Public Health of the University of Kinshasa.

\section{Conflicts of Interest}

The authors declare no conflicts of interest regarding the publication of this paper.

\section{References}

[1] World Health Organization (1996) Mother and Child File: Guide for Safe Motherhood. World Health Organization, Geneva, 110 p.

[2] Al-Qutob, R., Majali, S., Massad, D., Touka, O. and Schimidt, A.B. (2000) Perceptions of Health and Seeking Care: The Experience of Midlife Women in Ain Al-Basa, Jordan. International Quarterly of Community Health Education, 19, 163-178. https://doi.org/10.2190\%2F3NYX-9V5W-VEXJ-XWLU

[3] Afolabi, B.M., Ezedinachi, E., Oparah, S.K., Iwara, A. and Ogunwale, A. (2016) Perception of Obstetric Danger Signs among Womens Living on Coastline of the Atlantic Ocean in Rural Lagos, Nigeria. Journal of Public Health and Epidemiology, 8 , 1-11. https://doi.org/10.5897/JPHE2015.0765

[4] Shuval, J.T. (1981) The Contribution of Psychological and Social Phenomenal to an Understanding of the Etiology of Disease and Illness. Social Science \& Medicine. Part A: Medical Psychology \& Medical Sociology, 15, 337-342. https://doi.org/10.1016/0271-7123(81)90064-X

[5] Ministry of Public Health of the Democratic Republic of Congo (1998) Report on the Inventory of the Health Sector in the Democratic Republic of Congo; Health Profile of the Central Level, Provinces, Health Zones and Households. Ministry of Public Health of the Democratic Republic of Congo, Kinshasa, 239 p.

[6] Ministry of Public Health, Ministry of Planning and Reconstruction of the Democratic Republic of the Congo, United Nations Children's Fund and United States Agency for International Development (2002) National Survey on the Health Situation of Children and Women in the Democratic Republic of Congo (MICS2/2001) Analysis Report. Vol. I, Kinshasa. https://data.unicef.org/

[7] Ministry of Planning and Ministry of Public Health of the Democratic Republic of 
the Congo, United Nations Children's Fund, United States Agency for International Development, Department for International Development and World Bank (2007) Demographic and Health Survey in the Democratic Republic of Congo (EDS) synthesis Report. MPSMRM, MSP, and ICF International, Rockville.

[8] Ministry of Planning and Ministry of Public Health, United Nations Children's Fund, United States Agency for International Development, Department for International Development and World Bank (2014) Demographic and Health Survey in the Democratic Republic of Congo (EDS) Synthesis Report. MPSMRM, MSP, and ICF International, Rockville, 91-112.

[9] Wessel, H., Reitmaier, P., Dupret, A., Rocha, E., Cnattingius, S. and Bergström, S. (1999) Death among Women of Reproductive Age in Cape Verde: Causes and Avoidability. Acta Obstetricia et Gynecologica Scandinavica, 78, 225-232.

https://doi.org/10.1034/j.1600-0412.1999.780310.x

[10] Pictet, G., Le Cour, S., Mpele, P., Brouard, N. and Lallemant, M. (1998) Contribution of AIDS to the General Mortality in Central Africa: Evidence from a Morgue-Based Study in Brazzaville, Congo. AIDS, 12, 2217-2223. https://doi.org/10.1097/00002030-199816000-00018

[11] Mambu Nyangi Mondo, T., Malengreau, M., Kayembe Kalambayi, P. and Lapika Dimomfu, B. (2010) The Delays in Recourse and Care in Kinshasa in the Event of Severe Illness in Women of Childbearing Age. Revue d'Épidémiologie et de Santé Publique, 58, 189-196. https://doi.org/10.1016/j.respe.2010.02.107

[12] Urassa, E., Massawe, S., Lindmark, G. and Nystrom, L. (1997) Operational Factors Affecting Maternal Mortality in Tanzania. Health Policy and Planning, 12, 50-57. https://doi.org/10.1093/heapol/12.1.50

[13] Dongre, A.R., Deshmukh, P.R. and Garg, B.S. (2008) Danger Signs among Mothers in Rural Wardha. Indian Journal of Pediatrics, 75, 325-329.

https://doi.org/10.1007/s12098-008-0032-7

[14] Mays, N. and Pope, C. (1999) Quality in Qualitative Interviews in Health Care Research. In: Pope, C. and Mays, N., Eds., Qualitative Research in Health Care, BMJ Books, London, 89-101.

[15] De Nooijer, J., Lechner, L. and de Vries, H. (2001) A Qualitative Study on Detecting Cancer Symptoms and Seeking Medical Help: An Application of Andersen's Model of Total Patient Delay. Patient Education and Counseling, 42, 145-157. https://doi.org/10.1016/S0738-3991(00)00104-X

[16] Zajc, S. (2000) Analysis of Qualitative Data: Traditional Practice and Assisted by the NUD.IST Qualitative Research Software. Qualitative Researches, 21, 99-123.

[17] Kayembe Kalambayi, P. (2000) Report of the CAP Study on STDs/AIDS and on the Use of Condoms in Kinshasa. Kinshasa.

[18] Pembe, A.B., Urassa, D.P., Carlsted, A., Lindmark, G., Nyström, L. and Darj, E. (2009) Rural Tanzanian Women's Awareness of Danger Signs of Obstetric Complications. BMC Pregnancy and Childbirth, 9, Article No. 12. https://doi.org/10.1186/1471-2393-9-12

[19] Zola, I.K. (1966) Culture and Symptoms-An Analysis of Patients Presenting Complaints. American Sociological Review, 31, 615-630.

https://doi.org/10.2307/2091854

[20] Lapika, D., Kayembe, D., Noma, M. and Boussinesq, M. (2007) African Programme for Onchocerciasis Control (APOC): Sociological Study in Three Foci of Central Africa before the Implementation of Treatments with Ivermectin (Mectizan ${ }^{\circledast}$ ). Transactions of the Royal Society of Tropical Medicine and Hygiene, 101, 674-679. 
https://doi.org/10.1016/j.trstmh.2007.03.007

[21] Maine, D. and the Prevention of Maternal Mortality Network (1992) Barriers to Treatment of Obstetric Emergencies in Rural Communities of West Africa. Studies in Family Planning, 23, 279-291. https://doi.org/10.2307/1966526

[22] Thaddeus, S. and Maine, D. (1994) Too Far to Walk, Maternal Mortality in Context. Social Science \& Medicine, 38, 1091-1110. https://doi.org/10.1016/0277-9536(94)90226-7

[23] Bantebya Kyomuhendo, G. (2003) Low Use of Rural Maternity Services in Uganda Impact of Women's Status, Traditional Beliefs and Limited Resources. Reproductive Health Matters, 11, 16-26. https://doi.org/10.1016/S0968-8080(03)02176-1

[24] Mc Kinley, S., Moser, D.K. and Dracup, K. (2000) Treatment-Seeking Behavior for Acute Myocardial Infarction Symptoms in North America and Australia. Heart Lung, 29, 237-247. https://doi.org/10.1067/mhl.2000.106940

[25] Fawcus, S., Mbizvo, M., Lindmark, G. and Nystrom, L. (1996) A Community-Based Investigation of Avoidable Factors for Maternal Mortality in Zimbabwe. Studies in Family Planning, 29, 319-327. https://doi.org/10.2307/2138027

[26] Mehta, U., Durrheim, D.N., Blumberg, L., Donohue, S., Hansford, F., Mabuza, A., Kruger, P., Gumede, J.K., Immelman, E., Sanchez Canal, A., Huggo, J.J., Swart, G. and Barnes, K.I. (2007) Malaria Deaths as Sentinel Events to Monitor Healthcare Delivery and Antimalarial Drug Safety. Tropical Medicine and International Health, 12, 617-628. https://doi.org/10.1111/j.1365-3156.2007.01823.x

[27] Ekotarl, A., Ndom, P. and Sacks, A. (2007) A Study of Patients Who Appear with Far Advanced Cancer at Yaounde General Hospital, Cameroon, Africa. Psycho-Oncology, 16, 255-257. https://doi.org/10.1002/pon.1144

[28] Yimer, S., Bjune, G. and Alene, G. (2005) Diagnostic and Treatment Delay among Pulmonary Tuberculosis Patients in Ethiopia: A Cross Sectional Study. BMC Infectious Diseases, 5, Article No. 112. https://doi.org/10.1186/1471-2334-5-112

[29] Jaramillo, E. (1998) Pulmonary Tuberculosis and Health Seeking Behaviour: How to Get Delayed Diagnosis in Cali, Colombia. Tropical Medicine \& International Health, 3, 138-144. https://doi.org/10.1046/j.1365-3156.1998.00202.x

[30] Meyer-Weitz, A., Reddy, P., Weijts, W., Van den Borne, B. and Kok, G. (1998) The Socio-Cultural Contexts of Sexually Transmitted Diseases in South Africa: Implications for Health Education Programs. AIDS Care, 10, 39-55. https://doi.org/10.1080/09540129850124352

[31] Subedi, J. (1989) Modern Health Services and Health Care Behavior: A Survey in Kathmandu, Nepal. Journal of Health and Social Behavior, 30, 412-420. https://doi.org/10.2307/2136989

[32] Kennedy, J.C. and Olsson, K. (1996) Health Care Seeking Behaviour and Formal Integration: A Rural Mexican Case Study. Human Organization, 55, 41-46. https://doi.org/10.17730/humo.55.1.f376213mu8611573

[33] Awasthi, S., Verma, T. and Agarwal, M. (2006) Danger Signs of Neonatal Illnesses: Perception of Caregivers and Health Workers in North India. Bulletin of the World Health Organization, 84, 819-826. https://doi.org/10.2471/BLT.05.029207

[34] Urassa, E., Lindmark, G. and Nyström, L. (1995) Maternal Mortality in Dar es Salaam, Tanzania: Socio-Economic, Obstetric History and Accessibility of Health Care Factors. African Journal of Health Sciences, 2, 242-249.

[35] Ngondo à Pitsandenge, S. (2000) From Maternal Mortality in the Democratic Republic of Congo to the Dawn of the 3rd Millennium. Levels and Explanatory Factors. In: Congo-Afrique, R., Ed., Congo-Afrique: Economie-Culture-Vie Sociale, Kinshasa, 347. 